\title{
Disulfiram was effective in patients with cocaine abuse who were also methadone treated opioid addicts
}

Petrakis IL, Carroll KM, Nich C, et al. Disulfiram treatment for cocaine dependence in methadone-maintained opioid addicts. Addiction $2000 \mathrm{Feb}$;95:219-28.

\section{QUESTION: What is the effectiveness of disulfiram for cocaine dependence in patients who are also opioid addicts treated with methadone?}

\section{Design}

Randomised \{allocation concealed\}*杵, blinded (investigator and patient)†, placebo controlled trial with 12 weeks of follow up.

Setting

Urban substance abuse treatment centre in the US.

\section{Patients}

69 outpatients \{mean age 35 y, 53\% women, 74\% white |* with DSM-III-R criteria for cocaine dependence who were on methadone treatment and using cocaine frequently (defined as having $\geqslant 3$ urine toxicology screens positive for cocaine in the 1 mo before study). Exclusion criteria were psychotic or bipolar disorders, serious psychiatric symptoms, or contraindications to disulfiram use. Follow up was $75 \% .97 \%$ were included in the analysis.

\section{Intervention}

Patients were allocated to disulfiram, $250 \mathrm{mg} /$ day (dissolved directly in the methadone) $\{\mathrm{n}=37\}^{*}$, or placebo $\{\mathrm{n}=32\} *$ for 12 weeks. Patients used a mean methadone dose of $88.6 \mathrm{mg} /$ day, and were counselled about the risk of a disulfiram alcohol interaction and advised not to drink alcohol. During the study, patients adhered to a behavioural contract with a reduction of methadone dose for violations.

\section{Main outcome measures}

Frequency and quantity of cocaine, alcohol, and other substance use were self reported and verified by urine tests and breathalyser readings.

\section{Main results}

Patients who received disulfiram had a lower mean number of days of cocaine use than those who received placebo (5.0 $v 6.7 \mathrm{~d}, \mathrm{p}=0.04)$. Furthermore, those who received disulfiram had a greater mean decrease in quantity $(p=0.02)$ of cocaine use than those who received placebo; groups did not differ for alcohol or heroin use. The effectiveness of disulfiram did not differ in patients who did and did not use alcohol at baseline.

\section{Conclusion}

Disulfiram was effective in patients with cocaine abuse who were also methadone treated opioid addicts with or without comorbid alcohol abuse.
*Information provided by author.

$\dagger$ See glossary.

\section{COMMENTARY}

Cocaine use is increasingly prevalent among patients entering methadone maintenance treatment and is associated with poor treatment outcomes. ${ }^{1}$ This study by Petrakis $e t a l$ examines the effectiveness of disulfiram in reducing cocaine use in patients on methadone treatment. Unfortunately, because both placebo and disulfiram groups had large decreases in heroin, ethanol, and cocaine use, the largest treatment effect was not medication specific but because of enrolment in the study. Against the background of this decreasing use, the disulfiram group had a significant decrease in cocaine use over time compared with the placebo group despite the absolute level of use at the end of the trial being not dramatically different between the groups.

Adverse events were clearly an issue in the disulfiram group with 19\% dropping out of treatment because of medical or psychiatric side effects (compared with $0 \%$ in the placebo group). The clinical utility of disulfiram would have been easier to assess if the final cocaine outcomes had included all randomised participants rather than just those who completed treatment (ie, intention to treat analysis). Systematic monitoring of serum transaminases concentrations would also have been clinically important, given the high prevalence of viral hepatitis in this patient population, ${ }^{23}$ who therefore may also have an increased risk of disulfiram induced hepatoxicity. Finally, the generalisability of these findings to patients with primary cocaine dependence is not strong because methadone maintenance treatment is likely to enhance compliance with disulfiram. Overall, this study supports the need for further research on the potential utility of disulfiram in the management of treatment resistant cocaine use among methadone maintained patients.

David Marsh, MD Centre for Addiction and Mental Health Toronto, Ontario, Canada

1 Grella CE, Anglin MD, Wugalter SE. Cocaine and crack use and HIV risk behaviors among high-risk methadone maintenance clients. Drug Alcohol Depend 1995;37:15-21.

2 Levine OS, Vlahov D, Nelson KE. Epidemiology of hepatitis $B$ virus infections among injecting drug users: seroprevalence, risk factors, and viral interactions. Epidemiol Rev 1994;16:418-36.

3 Patti AM, Santi AL, Pompa MG, et al. Viral hepatitis and drugs: a continuing problem. Int J Epidemiol 1993;22:135-9. 